\title{
Acclimatization of Diatom Phaeodactylum Tricornutum to Long-Term Environmental Temperature and Light Intensity Changes
}

\author{
Edyta Warkiewicz $^{1,2}$, Agnieszka Strączek ${ }^{1}$, Dariusz Latowski ${ }^{1}$, Monika Bojko ${ }^{\text {* }}$ \\ ${ }^{1}$ Department of Plant Physiology and Biochemistry, Faculty of Biochemistry, Biophysics and Biotechnology, Jagiellonian \\ University, Gronostajowa 7, 30-387 Kraków, Poland \\ edyta.warkiewicz@gmail.com; agnieszka.straczek@op.pl; dariuszlatowski@gmail.com; \\ m.bojko@uj.edu.pl* corresponding author \\ ${ }^{2}$ Faculty of Chemistry, Central Magazine for Chemical Reagents and Waste, Jagiellonian University \\ Gronostajowa 2, 30-38 Kraków, Poland
}

\begin{abstract}
Diatoms are the dominant primary producers in a number of oceanographic settings that require high-nutrient and turbulent conditions. In addition, diatoms are sensitive indicator of environmental changes and small modifications of environment result in measurable changes in physiological and biochemical parameters. In the present study the model diatom species, Phaeodactylum tricornutum, was culturing for 6 months and the influence of two environmental factors i.e. temperature and light intensity, as the model of greenhouse effect, was tested. Two temperatures i.e. $12^{\circ} \mathrm{C}$ (low) and $20^{\circ} \mathrm{C}$ (moderate), and two light intensities i.e. $6 \mu \mathrm{Em}^{-2} \mathrm{~s}^{-1}$ (low) and $40 \mu \mathrm{Em}^{-2} \mathrm{~s}^{-1}$ (moderate) were selected. Three main growth phases of diatom cultures were observed. In the first phase (first two weeks) the optical density at $600 \mathrm{~nm}(\mathrm{OD})$ of cultures as well as photosynthetic activity measured as Fv/Fm parameter, increased under all experimental conditions. However, in third growth phase i.e. after four months of experiments, the decrease of OD was detected only at a higher temperature. Values of $\mathrm{Fv} / \mathrm{Fm}$ parameter showed that photosynthetic activity of $P$. tricornutum was more sensitive to light intensity than to growth temperature and lower photosynthetic activity was detected at moderate light intensity. In the third phase photosynthetic activity was also well correlated with chlorophyll a concentration and the carotenoid content. The obtained results indicated that the growth rate of diatom cultures depends on temperature whereas photosynthetic activity is more sensitive to light intensity. Low temperature as well as low light intensity were found to be better environmental conditions which ensure a sustainable and long-term growth of diatoms.
\end{abstract}

Keywords: Diatoms, Long-Term Acclimatization, Temperature, Light Intensity, Photosynthesis, Chlorophylls, Carotenoids.

\section{Introduction}

The warming planet surface and increasing ocean heat content may be the result of seasonal changes in temperature or changes related to the greenhouse effect. All changes in temperature and water warming can have an impact on water ecosystems. These changes strongly influence the photosynthetic activity of phytoplankton, which results in CO2 fixation and finally biomass production [1-4].

Diatoms (Heterokontophyta, Bacillariophyceae) are a major group of microalgae, the dominant primary producers in a number of freshwater, marine and oceanographic ecosystems that require high-nutrient and turbulent conditions. They fix 20 billion tons of carbon per year and are responsible for around 20\% of global net primary photosynthetic production [4-6]. In addition, diatoms are sensitive indicator of environmental changes and small modifications of environmental result in measurable changes in physiological and biochemical parameters [1,7].

Despite the ecological significance of diatoms, there is only few data about the effect of long-term temperature and light changes as well as about acclimatization mechanism to these environmental conditions. The purpose of the work was the analysis of parameters that may be used to monitor the physiological and biochemical state of diatoms during the long-term cultures in changing environmental conditions. The growth parameter, photosynthesis efficiency and content of the photosynthetic pigments were monitored at low and moderate temperature and two (low and moderate) light intensities. Both were applied as the model of climate warming under laboratory conditions. For the long-term study of diatoms adaptation mechanism to the environmental changes, the model marine diatom Phaeodactylum tricornutum was selected. 


\section{Materials and Methods}

The $P$. tricornutum CCAP/1055/1 strain, obtained from the Culture Collection of Algae and Proteoza at the Dunstaffnage Marine Laboratory, UK, was grown in standard culture conditions in $\mathrm{f} / 2$ medium [8], under photoperiod $10: 14 \mathrm{~h} \mathrm{D}: \mathrm{L}$. The diatom cultures were carried out in two white light intensities: at moderate light intensity of $40 \mu \mathrm{Em}^{-}$ ${ }^{2} \mathrm{~s}^{-1}$ in the standard culture bottles and low light intensity of $6 \mu \mathrm{Em}^{-2} \mathrm{~s}^{-1}$ in the amber culture bottles (Borosilicate Glass, SIMAX 2L) both at low $\left(12^{\circ} \mathrm{C}\right)$ and moderate temperature $\left(20^{\circ} \mathrm{C}\right)$ [9]. Cultures were shaken $(50 \mathrm{rpm} / \mathrm{min})$ during the light and dark phase to keep cells in suspension and maintain an optimal exchange of gas and nutrients as the model of sea water fluctuation. The samples were collected in the first hour of the light phase on first, $14^{\text {th }}, 58^{\text {th }}, 112^{\text {th }}, 140^{\text {th }}$ and $181^{\text {th }}$ day after inoculation.

Pigments for concentration analysis were extracted from $P$. tricornutum in medium $1((90 \% \mathrm{MeOH} / 10 \% 0.2 \mathrm{M}$ ammonium acetate), $10 \%$ ethyl acetate). After centrifugation supernatant was mixed with chloroform and water (in rate: 2:1:1), vortexed, centrifuged. Lower phase was collected, evaporated and dissolved in $90 \%$ acetone.

Concentration of the chlorophyll a was determined with a spectrophotometer and calculated by Jeffrey and Humphrey method [10]

After extraction in medium 1, pigments content in thylakoid membranes was analyzed on the Nucleosil column (ET 250/8/4, 300-5 C18, Maderey\&Nagel, Germany) by HPLC, applying a gradient according to Kraay [11].

Cell density was measured at $600 \mathrm{~nm}$ [12] with a spectrophotometer.

Photosynthetic efficiency was estimated by chlorophyll fluorescence measurement by Hansatech Pocket PEA (Waltz, Germany). Before measurements samples were collected and centrifuged to obtain pellet of the cells which were dark-adapted for 15 min [9]. Software generated Fo (minimum) and Fm (maximum) fluorescence values and calculated the optimum quantum yield Fv/Fm.

The average cell size was measured by LUNA ${ }^{\mathrm{TM}}$, an automated cell counter, that accurately measures the size by Bright Field cell counting mode.

The observation of diatom cell morphotype and photographic documentation were taken using Nicon Eclipse TE 200 microscope with a Nomarski DIC prism with a 60x objective. The microscope was equipped with Monochrome Camera [9].

Statistical calculations were performed with Statistica program. Means differences comparison among different treatments was done by Student's T or by Kruskala-Wallisa test.

\section{Results}

The effect of temperature and adaptation mechanisms of marine diatoms $P$. tricornutum and $T$. pseudonana, growing two weeks in batch cultures, was presented and discussed in our previous papers $[9,13]$. In this document we present the effect of temperature and light intensity on $P$. tricornutum growth, cells size and selected photosynthesis parameters, which were analyzed during six months. The survival and adaptation to the environment changes were monitored by analysis of parameters which provide information about physiological and biochemical conditions of diatom cultures.

The comparative analysis of growth kinetics, monitored by cell densities $\left(\mathrm{OD}_{600}\right)$ of $P$. tricornutum culture at low and moderate temperature and under two light conditions, was performed (Fig.1A). This parameter informs about biomass increase and biological condition of phytoplankton community [7]. The obtained results showed significant differences in diatom growth kinetics at different temperatures and light conditions. The growth of diatoms under both temperatures was characterized by three phases. First, the fastest and linear, observed during the first two weeks of culture showed no statistical significant differences in the growth kinetics between all tested cultures. The second phase with the high rate growth (where OD increased fast), observed between the second and the end of the $16^{\text {th }}$ week after the inoculation, was more intensive in cultures at a low temperature than at a moderate temperature. The rate growth in the third phase showed significant differences in the diatom growth kinetics at both temperatures $\left(12\right.$ and $\left.20^{\circ} \mathrm{C}\right)$. The growth rate at low temperature was slightly lower than in the $2^{\text {nd }}$ phase (especially at low light) while at moderate temperature the cell density remained almost at a constant level (at both light condition) during the last two months of measurements. Although no effect of temperature, either low or moderate, on growth rate of diatoms was observed during the first phase, in the second and third phases the growth rate was clearly limited at moderate temperature. 

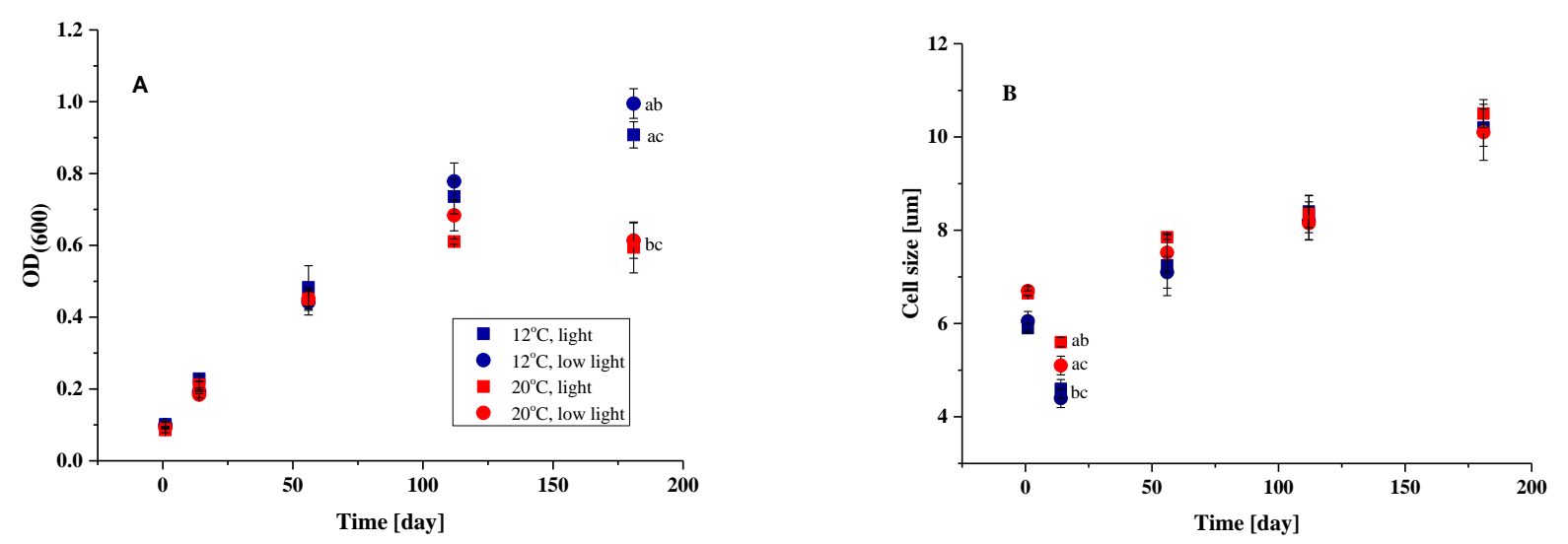

Fig. 1: The effect of temperature and light intensity on OD (A) and cell size (B) of P. tricornutum growing in batch culture. Presented values are the average of eight experiments ( \pm standard error), the common letters close to symbols of arithmetic medium show statistical differences between groups of different treatment at $\mathrm{p} \leq 0.02$ according to Student's $\mathrm{t}$-test.

In general, the small amount of information has been obtained about the effect of the environmental stressors on the diatom size and morphological characteristic of diatoms [7]. P. tricornutum has three morphotypes of cell: oval, fusiform and triradiate. In our diatom cultures the most dominant morphotype was fusiform (Fig. 2). The division of fusiform diatom cells reduces their final size $[14,15]$. Moreover, it was observed that cells size depend on the growth temperature. The size of cells at 23 to $25^{\circ} \mathrm{C}$ were larger than those that grew at lower temperature [16]. In the beginning of our experiment, the average cell size was larger at moderate than at low temperature. The intensive division of cells, in the first phase of culture growth, resulted in the decrease of cell size from 6 to $4.5 \mu \mathrm{m}$ at low temperature and from 6.8 to $5.5 \mu \mathrm{m}$ at moderate temperature (Fig.1B). Finally, the size increased up to $10.5 \mu \mathrm{m}$ in the second and third phases in correlation with OD changes, which confirms the slowdown of the cultures growth.

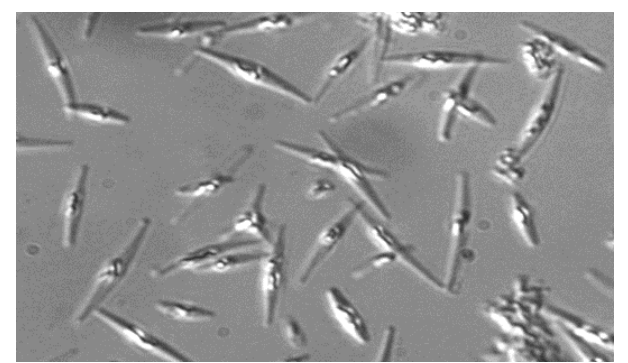

Fig. 2: P. tricornutum cells grown at $12^{\circ} \mathrm{C}$ under moderate light intensity, collected two weeks after inoculation. (Photo taken by Weronika Krzeszowiec).

The diatom chloroplasts are surrounded by four envelope membranes and thylakoids are arranged in groups of three [17]. The light harvesting system called fucoxanthin binding proteins (FCP), which function is to act as an antenna in photosynthesis, bind the high amount of fucoxanthin (an unique xanthophyll pigment) [18, 19] and additionally diadinoxanthin and diatoxanthin which are engaged in photoprotective mechanism known as diadinoxanthin cycle [20, 21]. FCP contain chlorophyll a (Chl a) and characteristic c1, c2 chlorophylls [18, 19, 22]. Photosynthesis can be used to monitor the community productivity and biological conditions [7]. The photosynthetic efficiency of photosystem II (Fv/Fm) is the most frequently used parameter to determine stress effects on diatoms photosynthesis condition [23]. The significant variability in $\mathrm{Fv} / \mathrm{Fm}$ parameter was observed in a long-term $P$. tricornutum culture at low and moderate temperature (Fig. $3 \mathrm{~A})$. In the first and the second phase of cultures growth, the level of Fv/Fm parameter was higher in diatoms at moderate than low temperature and was the highest after first two weeks of measurements under all tested growth conditions. However, later all cultures showed a slowly decrease in Fv/Fm values. After the four-month experiment, the Fv/Fm values of the culture were higher in low light than in the moderate and were the highest under low light and low temperature conditions. 

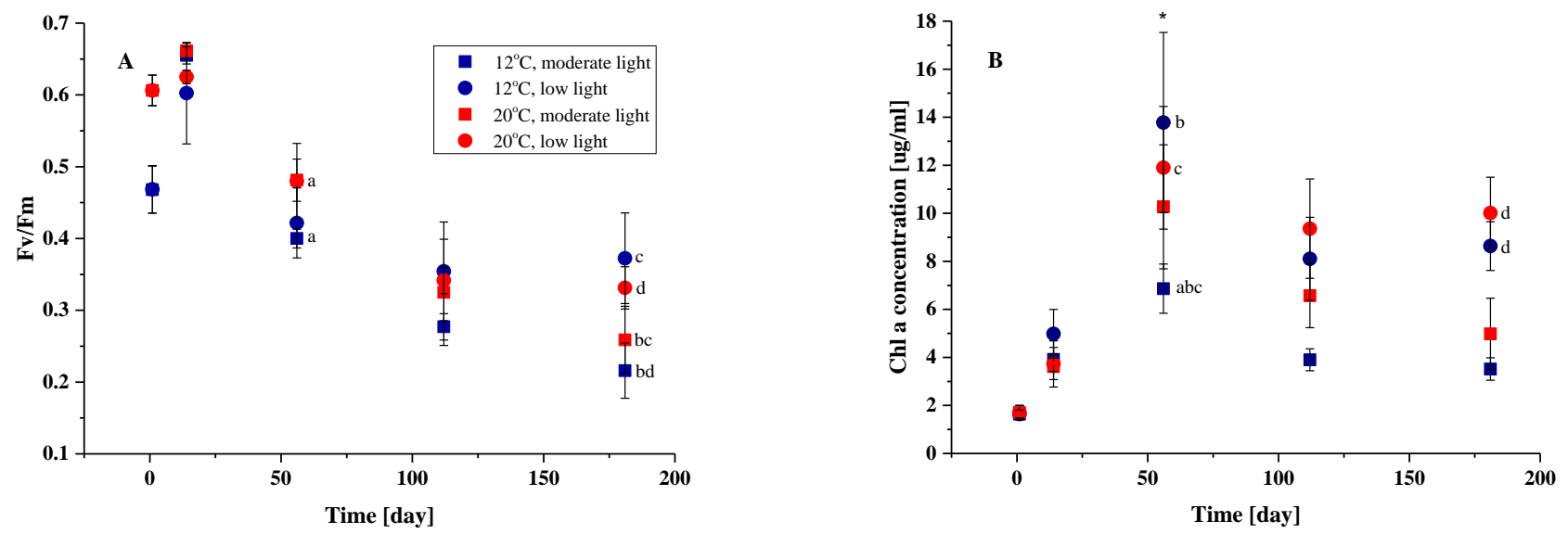

Fig. 3: The effect of temperature and light intensity on Fv/Fm (A) and chlorophyll a (Chl a) concentration (B) in P. tricornutum growing in batch culture. Presented values are the average of eight experiments ( \pm standard error), ), the common letters close to symbols of arithmetic medium show statistical differences between groups of different treatment at $\mathrm{p} \leq 0.0005$ according to Student's ttest or Kruskala-Wallisa test $(*)$.

The chlorophyll a concentration was increasing in correlation with OD values in the first phase (Fig. 3B). The significant differences in the chlorophyll a concentration were observed already in the second month of the cultures. Although the decrease of $\mathrm{Chl}$ a was observed at both temperatures, the highest Chl a concentration was detected at low light intensity. The changes in $\mathrm{Chl}$ a concentration may be the result of chlorophyll degradation, observed in diatoms processes involved in senescence and death of these photosynthetic cell [24].

The carotenoid content was calculated in comparison to all photosynthetic pigments (chlorophylls and carotenoids). The significant increase in carotenoid content was observed only in low light and at both temperatures (Fig. 5). The increase of carotenoid content, in the long time growth, may indicate the importance of the carotenoids function as well as differences in the changes associated with senescence processes occurring under various temperature and light conditions [24].

The changes of photosynthetic activity in the third phase, correlated with chlorophyll a concentration (Fig. 3B) and the carotenoid content changes (Fig 5).
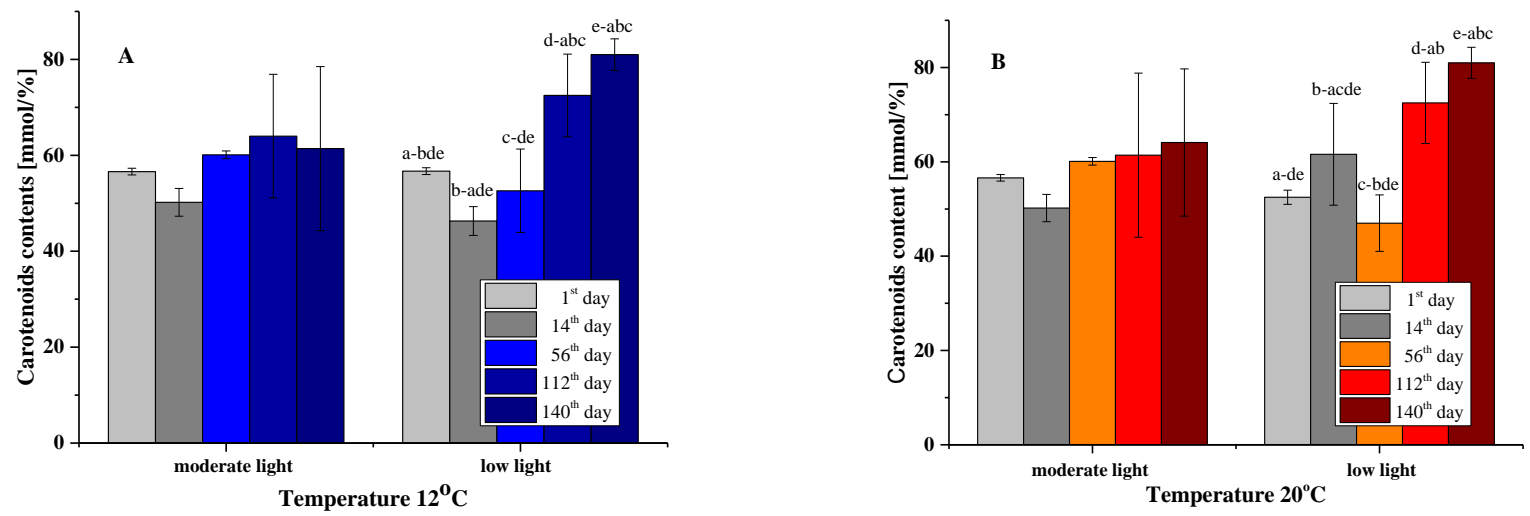

Fig. 4: The effect of light intensity on carotenoids content of $P$. tricornutum growing at low $\left(12^{\circ} \mathrm{C}\right)(\mathrm{A})$ and moderate $\left(20^{\circ} \mathrm{C}\right)$ (B) temperature in batch culture. Presented values are the average of six experiments ( \pm standard error). Error bars with common letters are significantly different at $\mathrm{p} \leq 0.05$ according to Student's t-test. 
Interpreting and predicting consequences (as a acclimatization or death of microalgae) of long-term biological changes, can be achieved using long-term collection of planktonic biota from multiyear time series and ecosystems [6]. In this paper we present results of the long-term effect of model parameters which help to interpret the physiological conditions of plankton in natural environment changes.

\section{Conclusion}

Light and temperature changes, which are important environmental factors, have impact on the physiological and biochemical processes in model diatom $P$. tricornutum. The obtained results present the diatoms adaptation strategies i.e. different proliferation, regulation of photosynthetic activity and changes in photosynthetic pigment content. Long-term monitoring of biochemical and physiological parameters shows that P. tricornutum prefer "cold-water and low-light" conditions.

\section{Acknowledgements}

We would like to thank Monika Olchawa-Pajor for her technical help and statistical analysis. This work was supported by KNOW-The Faculty of Biochemistry, Biophysics and Biotechnology of Jagiellonian University a partner of Leading National Research Centre (KNOW) supported by the minister of Science and Higher Education No. 35p/27/2016-2017 and A1/373.

\section{References}

[1] P. Snoeijs, K. Weckstrom, "Diatoms and environmental change in large brackish-water ecosystems," in The Diatoms: Applications for the Environmental and Earth Science, J. P. Smol and E. F. Stoermer ed., Cambridge University Press, 2010, pp. 287-308.

[2] S. Levitus, J. I. Antonov, T. P. Buyer, C. Stephens, "Warming of the World Ocean," Science, vol. 287, no. 5461, pp. 2225-2230, 2000.

[3] J. R. Toggweiler, J. Russel, “Ocean circulation in warming climate," Nature, vol. 451, no. 17, pp. 286-288, 2008.

[4] E. V. Armbrust, "The life of diatoms in the world's oceans," Nature, vol. 459, no. 14, pp. 185-192, 2009.

[5] P. G. Falkowski, R. T., Barber V. Smetacek, "Biochemical controls and feedbacks on ocean primary production," Science, vol. 281, pp. 200-206, 1998.

[6] O. E. Romero, L. K. Armand, "marine diatoms as indicators of changes in oceanographic condition" in The Diatoms: Applications for the Environmental and Earth Science, J. P. Smol and E. F. Stoermer ed., Cambridge University Press, 2010, pp. 373-400.

[7] R. J. Stevenson, Y. Pan, H. van Dam, Assessing environmental condition in rivers and streams with diatoms" in The Diatoms: Applications for the Environmental and Earth Science, J. P. Smol and E. F. Stoermer ed., Cambridge University Press, 2010, pp. 57-85.

[8] R. R. L. Guillard, J. H. Ryther, "Studies of marine planktonic diatoms. I. Cyclotella nana Hustedt and Detonula confervacea Cleve," Can. J. Microbiol., vol. 8, pp. 229-239, 1962.

[9] M. Bojko, K. Brzostowska, P. Kuczyńska, D. Latowski, M. Olchawa-Pajor, W. Krzeszowiec, A. Waloszek, K. Strzałka, "Temperature effect on growth, and selected parameters of Phaeodactylum tricornutum in batch cultures," Acta Biochimica Polonica, vol. 60, no. 4, pp. 861-864, 2013.

[10] S. W. Jeffrey, G. F. Humphre, "New spectrophotometric equation for determining chlorophyll a, b, c1 and c2," Biochem. Physiol. Pflanz., vol. 167, pp. 194-204, 1975.

[11] G. W. Kraay, M. Zapata, M. J. W. Veldhuis, ,Separation of chlorophylls $\mathrm{c}_{\mathrm{c}}, \mathrm{c}_{2}$, and $\mathrm{c}_{3}$ of marine phytoplankton by reversed-phase-C18-high-performance liquid chromatography," J. Phycol. vol.28, pp. 708-7812, 1992.

[12] W. Yongmanitchai, P. Ward, "Growth of Omega-3 fatty acid production by Phaeodactylum tricornutum under different culture conditions," App.l Environ. Micriobiol., vol. 57, pp. 419-425,1991.

[13] M. Bojko, M. Olchawa-Pajor, M. Chyc, R. Goss, S. Schaller-Laudel, D. Latowskil, "Acclimatization of Thalassiosira pseudonana photosynthetic membranes to environmental temperature changes," Proceedings of the 3rd World Congress on New Technologies (NewTech'17) Rome, Italy, paper no. ICEPR 120, 2017. doi: 10.11159/icepr17.120, 2017. 
[14] M. A. Borowitzka, B. E. Volcani, "The polymorphic diatoPhaeodactylum tricornutum: ultrastructure of its morphotypes," J. Phycol., vol. 14, pp. 10-21, 1978.

[15] A. D. Martino, A. Bartual, A. Willis, A. Meichenin, B. Villazán, U. Maheswari, C. Bowler, "Physiological and molecular evidence that environmental changes elicit morphological interconversion in model diatom Phaeodactylum tricornutum. Protist, vol. 162, pp. 462-481, 2011.

[16] M. W., Fawley, "Effects of light intensity and temperature interaction on growth of Pheodactylum tricornutum (Bacillariophyceae)," J. Phycol. vol. 20, pp. 67-72, 1984.

[17] A. M. Pyszniak, S. P. Gibbs, "Immunocytochemical localization of photosystem I and the fucoxanthin-chlorophyll a/c light-harvesting complex in the diatom Pheodactylum tricornutum," Protoplasma, vol. 166, pp. 208-2017, 1992.

[18] B. Lepetit, D. Volke M. Gilbert, Ch. Wilhelm, R. Goss, "Evidence for the existence of one antenna-associated, lipiddissolved and two protein-bound pools of diadinoxanthin cycle pigments in diatoms," Plant Physiol., vol. 154, pp. 1905-1920, 2010.

[19] B. Lepetit, R. Goss, T. Jacob, Ch. Wilhelm, "Molecular dynamics of the diatom thylakoid membrane under different light condition," Photosynth. Res., vol. 111, pp. 245-257, 2012.

[20] R. Goss, B. Lepetit, "Biodiversity of NPQ," J. of plant Physiol., vol. 172, pp. 13-32, 2015.

[21] R. Goss, T. Jakob, "Regulation and function of xanthophyll cycle-dependent photoprotection in algae," Photosynth Res. vol. 106, pp. 103-122, 2010.

[22] L. Premvardhan, B. Robert, A. Beer, C. Büchel, "Pigment organization in fucoxanthin chlorophyll a/c(2) proteins (FCP) based on resonance Raman spectroscopy and sequence analysis," Biochim. Biophys. Acta, vol. 1797, pp. 1647$1656,2010$.

[23] N. Domingues, A. R. Matos, J. M. de Silva, P. Cartaxana, "Response of diatom Pheodactylum tricornutum to photooxidative stress resulting from high light exposure," PLOS one, vol. 7, no. 6, e38162, 2012.

[24] J. W. Louda, L. L. Earl, W. Baker, "Senescence and death related alteration of chlorophylls and carotenoids in marine phytoplankton,” Organic Geochem., vol. 33, pp. 1635-1653, 2002. 